\title{
The processes of DNA replication and the shortening of the telomere are influenced by the action of the magnetic field
}

\author{
Rojeab Adnan Yousif \\ Electrical and Electronic Department, London College UCK, London, U.K. \\ Email address: \\ rojeab@btinternet.com
}

To cite this article:

Rojeab Adnan Yousif. The Processes of DNA Replication and the Shortening of the Telomere are Influenced by the Action of the Magnetic Field. International Journal of Genetics and Genomics. Vol. 2, No. 6, 2014, pp. 114-120. doi: 10.11648/j.ijgg.20140206.13

\begin{abstract}
This hypothesis research work shows that the induction and the remanent phenomena of the magnetic properties govern the mechanism of the processes of DNA replication and the shortening of the telomere. The solenoid-like formation of each parental DNA strand, which exists at the initial stage of the replication process, enables an electric charge transformation through the strand to produce a magnetic field. The magnetic field, in turn, induces the surrounding medium to form a new (replicated) strand by a remanent magnetization. Through the remanent [residual] magnetization process, the replicated strand possesses a similar information pattern to that of the parental strand. In the same process, the remanent amount of magnetization forms the medium in which it has less of both repetitive and pattern magnetization than that of the parental strand, therefore the replicated strand shows a shortening in the length of its telomeres.
\end{abstract}

Keywords: DNA Replication, Magnetic Properties, Residual Magnetization, Shortening of the Telomere

\section{Introduction}

Biological and chemical processes are playing an important role on the function of the DNA. Also, it is shown by variety of literatures that the electrical and magnetism are playing an important role on the function of the cell and that of DNA process.

Therefore, a general explanation to the function of electrical and magnetism is important in this matter.

\section{Literature Review on: Structure, Replication and Electrical Properties of the DNA}

The most important property of DNA for a bio-molecular engineer is its ability to self- assemble, which makes it possible to produce nano-structures [1]. Also, it is the best nano-wire known in existence, and it self-assembles, selfreplicates and can adopt various states and configurations [2].

DNA is made up of double helix strands, which are two coiling structures spliced onto each other in an anti-parallel manner.

However, a telomere is a special structure composed of DNA-protein present at the end of a chromosome in a nongenetic way. Telomeres serve as protective caps to prevent a chromosomes end to end fusion, and allow complete replication.

The structure of the telomere is a repetitive DNA sequence TTAGGG, of base pairs, and in human consist of chemical clusters. The length of a telomere has frequently been used as a means to predict the life span of cells. But itself can be poor indicator of aging or cell viability [3].

Every time a chromosome is replicated, a bit of its telomere is lost. When the length of the telomere reaches a certain shortness (critically small), the chromosome reaches a critical length and this situation leads to no more replication of the chromosome in the nucleus of cells. This leads, in turn, to the cell becoming old and no longer producing new cells. The cell is then said to be die by a process called apoptosis.

The reason behind the cessation of division is that some of the essential genes are lost from the daughter chromosome if the division is continued while the telomere is at critical length, and the result is to form a different structure and function of DNA from that of the parental one.

Many research works have been carried out concerning with the DNA replication. King [4] has shown that the precise nature of the origins of DNA replication in higher eukaryotic organisms is unclear. Also, Health Encyclopedia [5] has explained that the separation of the two strands of a DNA, in the replication process, is followed by the automatic formation of complementary strands on each of the separated strands. 
I think that the mechanism of these functions should not only be based on the biological and chemical aspects, but also the effect of electrical and magnetic phenomenon which has not been much addressed in scientific literature yet, have to be considered since the magnetic and electric effects have a great inheriting importance.

\section{Action of Magnetic Field}

A magnetic field can be produced by a current-carrying conductor. The magnetic field produced surrounds the conductor and, then, the outside medium is influenced by the effect of this magnetic field. A magnetic field intensity, $\mathrm{H}$, [Ampere / meter] acts as a magnetizing force to induce magnetization in the medium of surrounding magnetic materials, where the magnetic materials will possess a magnetic flux density, B [in Tesla].

The relationship between the applied magnetic field intensity, $\mathrm{H}$, and the induced magnetic flux density, $\mathrm{B}$, in the medium is giving by:

$$
\mathrm{B}=\mu \mathrm{H}
$$

where $\mu$ represents the permeability of the medium, as the medium been induced by the flux density $\mathrm{B}$ when $\mathrm{H}$ is applied.

The variation of $\mathrm{B}$ with $\mathrm{H}$ is nonlinear, and can be represented by the magnetization curve of Fig. 1.

When a non-magnetized magnetic material (as a medium) is exposed to a source of varied magnetic field, $\mathrm{H}$, a different amount of magnetic induction, $\mathrm{B}$, is applied to the material. As an increase in the magnetizing force, [magnetic field] is applied, the medium will possess an increase in its induced magnetization toward its saturation value, $B_{\text {sat, }}$. When the magnetizing force, $\mathrm{H}$, is reduced to zero (or absent), the induced magnetization of the medium is reduced as well, but it will not return to zero, as it lags behind the magnetizing force of the field [6].

Therefore, the induced magnetization of the medium is the amount of magnetization which is residual (or remanent) in the medium, in the absence of the source.

This is represented by the remanent magnetic flux density, $B_{r}$, which is usually less than that of the saturation flux density, $\mathrm{B}_{\text {sat }}$.

Induced magnetization in the medium has two main properties:

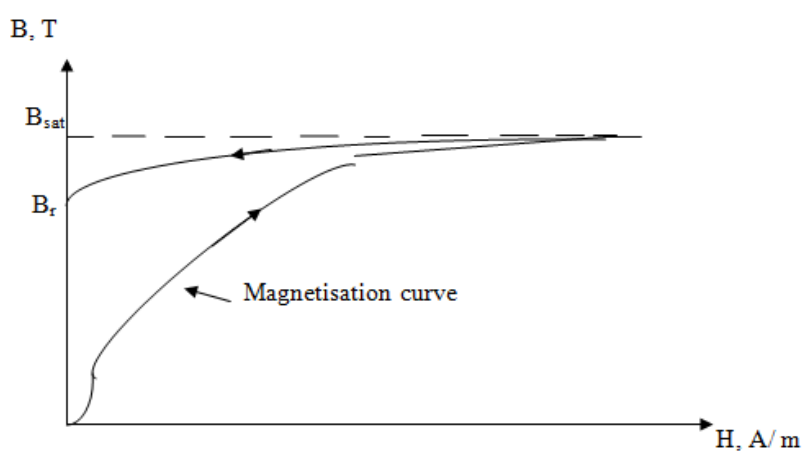

Fig. 1. $B$ - $H$ variation, and the residual flux density, $B_{r}$.
1- Showing a similar repetition analogue to the patterns of the applied magnetizing force (magnetic field), where the particles of the medium are magnetically aligned by the effect of the magnetizing field.

2- The value of the magnetic strength, in the induced medium, will be less than that of its maximum value, since the medium loses some of its induced magnetism by the absence of the magnetizing force.

Magnetic phenomena may be used to explain important aspects of cell's functions, such as that of the cause of DNA replication and the shortening of the telomere.

Therefore, this hypothesis work is concerned to explain the cause of the fundamental process of DNA replication.

As it is known that DNA replication involves the separation of the two strands over a short distance, followed by the automatic formation of complementary strands on each of the separated strands. The parental double helix strands of DNA, which are coiled and spliced together, are unfolded and held apart.

DNA does not split along its whole length at one time. Many short loops of separated DNA form simultaneously and the replication occurs in these loops [5]. Splitting the two strands apart starts at replication regions which are rich in adenine-thymine base pairs. The reason for the separation of the strands is to expose the bases so that new nucleotides partners can bond to each of the parental strands.

When the unfolding process is formed, each parental strand is shaped a solenoid- like. It will then, go on to replication. Each of the two strands in the original DNA acts as a template against which two new strands are built [7].

The surrounding environment to the parental strands is various types of amino acids, which are mainly a protein. The amino acids prepare the environment for the process of replication.

It is known that DNA has negative charges distributed along its length [8]. This interesting structure has led many scientists to carry out detailed research on many of its electrical aspects; almost $600-\mathrm{nm}$ ropes of DNA were studied by Fink [9] and were found to be good conductors.

This was confirmed by other workers [10], who have found that DNA can act as a molecular wire, with the phosphate bonds in the backbone acting as tunnel junctions allowing electrons to move along.

Even though the electrical conductivity of DNA is imperfect, it is of vital importance to the cell since the cell uses electrons through the charge transfer process to repair damaged bonds [11].

Further beneficial of DNA is to exhibit a property which is similar to the function of some electronic devices. Researchers [12] have shown that a 10.4- nm - long, double stranded poly (G) - poly (C) DNA molecules that is connected to two metal nano-electrodes, possesses electrical characteristics similar to that of semiconductors. The current measured was reported to be negligible up to threshold voltage which was followed by a sharp rise in the current.

This result shows similarity to the behavior of an electronic diode. 
The other example of the electrical domain of DNA was noticed when a team of researchers [13], while exploring the potential of DNA as an electric wire, have found that guanine is the base with the lowest oxidation potential which loses an electron during oxidative stress and becomes positively charged. This positive charge does not stay at the base where it was formed, but keeps moving along G-rich sequences.

Regarding the current that can be included in DNA, Fink and Shonenberger [10], were the first to measure current through DNA. They have reported direct measurements of electrical current as a function of the potential applied across a few DNA molecules associated into single ropes of at least $600 \mathrm{~nm}$ long, which has indicated efficient conduction through the ropes.

They have also found that the resistivity values derived from these measurements were comparable to those of conducting polymers, and have indicated that DNA transports electrical current as efficiently as a good semiconductor.

Similarly, the researchers have measured current verses voltage (I-V) characteristics of the DNA ropes to obtain upper values for their resistivity. The voltage applied to the manipulation- tip was continuously swept and the current was monitored with an electrometer.

These currents must be attributed to the current through the molecules as they are in vacuum. The researchers, also, indicated that, although their experiments established that DNA molecules are conductors, the mechanism allowing the transport of charges remain unclear.

Electrical conductance of DNA molecules was studied [14] theoretically by adopted the tight binding small polaron model to calculate the molecular conductance both in a quasi -coherent and the incoherent regime. In the experiment of the poly (d A) - poly (d T) and the poly (d G) - poly (d C) DNA molecules, a unique current - voltage (I -V) curve:

[I $\alpha \sinh (\mathrm{bV})]$, was observed, where coefficient $\mathrm{b}$ is the temperature dependences.

Besides the temperature dependence of $b$, the length dependences of the electric current in the two regimes were derived.

A model of electric charge travelling on DNA molecules is demonstrated by Yi and Orland [15]. Their research was carried out to elucidate the effects of interference on electronic properties of single stranded DNA or RNA molecules.

They showed that in double stranded DNA, base which pairs wind about the helical axis can be modelled as electrons travelling on a twisted ladder- like structure. They also stated that, when a magnetic flux is present, the current amplitude can be modulated.

Authors [16] work on direct measurement of electrical transport through single DNA molecules have stated that: seemingly contradicting results raised a debate over the ability of DNA to transport charge and the nature of the conduction mechanisms through it.

They confirm that, they develop an experimental approach for measuring current through DNA molecules, chemically connected on both ends to a metal substrate and to a gold nano-particle, by using a conductive atomic force microscope. Many samples could be made because of the experimental approach adopted here, which enabled them to obtain reproducible results with various samples, conditions, and measurement methods.

They presented multileveled evidence for charge transport through 26 - bp - long ds DNA of a complex sequence, characterized by s- shaped current voltage curves that show currents greater than $220 \mathrm{n}$ A at $2 \mathrm{~V}$.

They stated that significant observation implies that a coherent or band transport mechanisms takes over for bias potentials leading to high currents of greater than $1 \mathrm{n} \mathrm{A}$.

More evidence for electrical transport, and that of current voltage characteristics, also for a semiconductor applicable of DNA strands were studied.

In their research [17], gold-DNA-gold nanoparticle (GNP) complexes were studied by scanning tunnelling microscopy (STM). I-V characteristics were collected from distinct positions of gold nanoparticle clusters and qualitatively explained by a tip- surface configuration model. Doublestranded DNA (ds DNA) of poly (dT)-poly(dA) was found to be slightly n-type semiconductor by theoretical simulation of the S-shaped I-V curves employing the Landauer formalism.

Research by Takagi et al [18] showed that, DNA electronic devices were prepared on silicon-based three-terminal electrodes. Both ends of DNA molecules (400 bp long, mixed sequences) were bridged via chemical bonds between the source-drain nano-gap $(120 \mathrm{~nm})$ electrodes. S-Shaped I-V curves were obtained and the electric current can be modulated by the gate voltage. The DNA molecules act as semiconducting p-type nanowires in the three-terminal device.

Electrical charges transfer on the DNA system are investigated by some researchers. Barton et al [19] in their examination of much longer charge-transport distance on DNA, they concluded that guanines are oxidized as a result of DNA- mediate charge transport over a significant distance (e.g. 200 Angstrom).

Also, the researchers found that, although long- range charge transfer is dependent on distance, it appears to be modulated by intervening sequence- dependent dynamics.

Barton et al stated that, single electrons can shoot far enough along DNA to influence genes activity. The electrical signals might help to switch genes that are far apart on and off. Also, the researchers stated that, it is a way of transmitting chemical information over a long distance that's dependent on a DNA sequence.

Montagnier et al [20] in their research on the capacity of some bacterial DNA sequences to induce electromagnetic waves at high aqueous dilutions, have found that genomic DNA may be able to send spook electromagnetic imprint of itself into distant cells and fluids.

Another research by Montagnier et al [21] on an acting of DNA magnetic on molecules of water, the researchers found that some bacterial and viral DNA sequences to induce low frequency electromagnetic waves in high aqueous dilutions. They, also, explained this phenomenon appears to be 
triggered by the ambient electromagnetic background of very low frequency.

While the mapping of the DNA process has opened up a great diversity of opinion, researchers world-wide intended to continue on a wider scale concerning its properties and functions and therefore an intense exploration of the biological and electrical properties and functions of DNA is taking place. The electrical potential of DNA and its nanoscale have given researchers a great hope to investigate and exploit the properties of DNA in an attempt to build nanoelectric technology; A-T and C-G base pairs combination aim to replace the semiconductor devices.

\section{Magnetic Devices}

Practical magnetic applications can be expressed by using magnetic devices, such as solenoid, which can act to produce an induced magnetisation in the surrounding medium.

\subsection{The Solenoid}

A solenoid carrying a current acts like a bar magnet. The effect of the magnetic field of the solenoid forms a pattern of a separated magnetic lines of flux. Each magnetic line is surrounding the solenoid from its inside to outside, where this pattern of the field is affecting the outside medium to induce magnetization in the medium. The formation of the magnetic lines is representing the direction of the north and the south magnetic poles of the solenoid. These phenomena are acting in a similar way to that of a bar magnet [22 ].

Therefore, while the surrounding medium is magnetically induced by the magnetic field intensity, $\mathrm{H}$, of the solenoid, the medium will possess a pattern formation which is similar to that of the applied magnetic field.

The induced and remanent magnetic phenomena are of a practical property and are widely applied in many technological aspects, such as that of the magnetic recording process which is used in various devices.

\subsection{Magnetic Recording Procedure}

Magnetic recording is a method used to store, (called "write"), information on a magnetic medium, by a magnetic field signal. The information occurs on the medium in the form of spatially separated regions of varying magnetization.

In the procedure of the magnetic recorder device, a magnetic tape is used as the storage medium.

In a magnetic recording device, the recording transducer or "head" converts an electrical signal to a magnetic signal (field). This field is applied to form a pattern of magnetization in the tiny magnetic particles of the tape (medium). The magnetic field of the recording head has the effect of altering the magnetic direction of the tiny particles on the tape so that these particles align their magnetic domains with the applied magnetic field. The pattern of the residual magnetization on the tape thus stores the representation of the signal of the applied field [23].

So the magnetic particles of a tape are induced by the affect of the magnetic signal of the recording head as they pass by a gap in the head.

As the tape passes against the position of the acting magnetic field of the recording head, different regions of the tape are magnetically induced in a magnetic spatial pattern corresponding to the acting field.

When a region of the tape is away from the acting magnetic field, a remanent magnetization is retained in that region. This region, through its remanent magnetization, possesses a similar pattern of magnetic information as that of the acting field of the head, but it will have less strength of magnetization.

This means that the region keeps the similar manner of the particular information in the head at that time when it has passed against the acting field.

The fixing process of the retained magnetic pattern in the medium depends on the magnetic coercive of the medium. The medium of a higher coercive is the more able to fix its magnetization when the applied magnetic field is removed.

The recorded magnetic tape can be used as a source to store its magnetic information on a second (i.e. a new) magnetic tape. The second magnetic tape will also possess a remanent magnetization, by the magnetic induction of the first tape (as a source). The amount of the magnetization, (the information) of the second tape, therefore, will be less than that of the first one.

This process can be continued when the second magnetic tape is used as a source to record its information on a third magnetic tape, and so on. As the process is continued, a situation will occur when a certain generation of magnetic tape will possess a very small amount of information, (magnetization), which is practically recorded as null information.

Similar results can be shown on the process of photocopier machine action. A brief of the items and function of the machine is that: developer of the machine is usually of magnetic ferrite. This developer acts as a magnetic / electrical carrier which allows the toner to be carried from the magnetic developer roller onto the electrically charged sensitive drum.

A thin wire(s) housed in a lightweight metal slide framework that electrical attracts the image / toner formed on to the drum onto the copy paper.

It can be shown that a first copy from an original [source] document is approximately similar to the original. If this copy is, now, used as a source for new copies, then a second generation copy [which is a copy from a first generation copy], is going to be less clear comparing with original document. Again, if the second generation copy is used to produce a third generation copy, and the third one is used to produce a fourth generation copy, and so on. Then each generation of a copy will be far to be clear in comparison with that of the original document. So, then, the $n^{\text {th }}$ copy generation will be like a poor paper, approximately has no any information.

This is again it is the action of magnetic residual phenomenon during the copy process. 
The fundamental idea applied in the magnetic recorder is similar to that used in every-day equipment such as sound recording (in an audio cassette), TV pictures (in a video cassette), data in the form of coded numbers and letters (in the memory of a computer disk).

\section{Discussion}

The initial process of the replication of a DNA strand is, as is mentioned above, initiated when the parental double helix strands of the DNA are unfolded and held apart, from their splice together. The result of this process forms each strand into a solenoid-like shape.

Also, as is shown in the literatures, the DNA molecule is an electrically conducting and an electrically charged material, through which electrical charges may be carried. It may be shown that these travel charges, along the solenoidlike strand of the DNA, form a sort of a nano-electric current. This current, in turn, produces a magnetic field which possesses a signal of a similar pattern to that of the electric current.

A magnetic field of the whole structure of the DNA molecule encounters difficulty to be detected, although some measurements have shown no net magnetic field. This result does not mean that the component constructions of DNA have no magnetic field.

I believe that each single strand of the DNA may be a source of a magnetic field, which is produced by the currentlike charges travelling along the system of the strand.

In an attempt to show the existence of a magnetic field on each single strand of a DNA molecule rather than on the whole structure of the DNA, I believe that it is important to emphasis on the situation of the parental DNA strand at the initial procedure before the completion of the replication process.

This procedure is as the following:

The parental strands, which are coiled together, are unfolded and held apart. The result of this procedure is going to occur the vital effect of the magnetic field's strength of each single strand, which means that each single strand can clearly show the effect of its magnetic strength.

By viewing the anti-parallel spliced direction of helical double strands in the structure of the DNA molecule, it can be understood that the signal effect of the magnetic field strength of the two strands will cancel each other out.

Therefore, this situation can lead to preventing the spliced double helix stranded, in the structure of the DNA molecule, to form any strength of magnetization. This would leave the whole structure of the DNA molecule at zero net magnetization. Clearly, the result of this process would not allow the whole spliced structure of the DNA molecule to share in any replication function.

Accordingly, a surrounding medium could be magnetically induced by the action of the magnetic field of each parental strand individually, (after the double stranded are unfolded).

In addition, it has been shown (6), that the double helix stranded in replication of DNA does not split along its whole length at one time, but in steps.

This means that the magnetic field intensity of each single parental strand increases gradually against the surrounding medium. This process could lead, also, to a gradual increase in the magnetic induction in the medium towards its saturation magnetic flux density, $\mathrm{B}_{\mathrm{sat}}$. It is a procedure which is analogous to that of the induced magnetization effect, as it is explained earlier.

These procedures, in turn, may allow the medium to possess a remanent magnetization. This means that the medium is formed its magnetic pattern structure analogous to that of the signal magnetic effect of the parental strand. Where the magnetization, as it is known, is the acting information which is induced in the medium by the parental individual strand.

This is a magnetic information process in the medium. It is similar, in its phenomenon, to that occurs in the photo copier machine action and in the magnetic recording process. In the magnetic recording process the tape recording medium is magnetically induced to form a similar pattern to that of the main acting magnetic source signal.

Now, each separated single parental strand is, therefore, acting by its induces magnetic field strength on the amino acid surrounding medium, in the nucleus.

Therefore, this medium is induced to form molecular structure analogous to that of the individual parental strand.

Accordingly, the main result shows that, through the replication process, each separated parental strand is acting by its magnetization, and, then, it will induce the pattern of its magnetic information in the surrounding medium.

The magnetically induced medium, which will possess the remanent magnetization [by the strand's magnetic field], may show two important characteristics:

1- The retention, through the remanent magnetization, of an analogue pattern construction is representing the magnetic formation as that of the parental strand. Where this is the result of the magnetic induction in the medium.

2- There will be a reduction in the strength of the magnetization occurs in the surrounding medium compare to that of the full repetition of the magnetic pattern of the parental strand. This reduction is due to the fact that the induced surrounding medium is formed by the remanent magnetization, $B_{r}$, which is less in magnetization strength than that of the parental strand (as a magnetic source). As that shown in Fig. 1, where, $B_{\text {sat }}$ represents the magnetization strength [magnetic flux density] of the source, which is as that of parental strand.

Therefore, it can be shown that:

A) The constructive formation of the surrounding medium molecules will be arranged similar to that of the single parental strand. This means that an induced (new) DNA strand is formed, which is a replicated strand by magnetic induction process.

B) The reduction of full repetition of the magnetic pattern, in the surrounding medium, may lead to replicate a less number of DNA bases. The lack in the number of DNA bases may be in such an order to form the telomere parts, as they 
are positioned at the terminals of a daughter strand, where the magnetic action is less comparing to other parts of the strand.

This result may show that the length of the replicated telomere is reduced in the comparison with the telomere of the parental strand.

The replication process, as is known, will lead to nucleus division, and the daughter chromosome will possess less length, (i.e. shortening), in its telomeres compared with the parental chromosome. This division sequence ends, after a certain number of nuclear divisions, in forming a critical shortening in the length of the telomere. The result may indicate that the nucleus, and then the cell itself, will not be able to divide again.

It can be realized that the fundamental DNA replication process may be shown as analogous to that of the magnetic recording process. The magnetic recording process indicates a series of reductions in the amount of magnetic information in the process of recorded tapes. Some reduction in magnetic information will occur in each later recorded tape generation, when it is magnetized by the act of the magnetic induction of the upper generation tape.

I believe that an important issue may be developed from the method of the DNA replication, as long as it is indicated that the replication process is performed by the magnetic induction action of the separated single strand only, which is as the follow:

The original formation of the two spliced strands forming a molecule of DNA is of importance matter, because the implication of this formation is to prevent the DNA as a whole molecule, [i.e. as one entity], from acting in replication. Where instead, each separated single strand is individually involved in the replication process, which the right life process.

If DNA molecule was able to act in the replication as a one complete structure, then the replication process would be continued without any interval stages. This would keep the nucleus packed with a huge number of chromosomes, which will change the whole of life.

\section{Conclusion}

Electromagnetic action is a fundamental source for the processes of both DNA replication and the shortening of the telomere. Magnetic field, as a result of the moving electrical charges along the DNA strands, is usually playing an important role to originate and construct the formation of the DNA daughter strands from the amino acid of the nucleus of the cell. The functions of the other factors as that of the enzymes hormones telomeras and others, are important for the replication of the DNA and shortening of the telomere, but the functions of these factors are governed by the action of the strand's magnetic field.

\section{Suggestion}

Apply the result of this hypothesis may lead to understand and treat the cancer, as I believe that the electric and magnetic phenomena are concerning with the cancer.

\section{References}

[1] Winfree, E., .Liu, F., Wenzler, L.A., and Seeman, N.C.(1998). Design and self-assembly of two dimensional DNA crystals. Nature, 394, 539-544.

[2] Bachtold, A., Hadley, P., Nakanishi, T. and Dekker, C. (2001). Logic circuits with carbon nanotube transistors. Science, 294, pp. $1317-1320$.

[3] Blackburn, E. H. (2000). Telomere states and cell fates, Nature 408, 53-56.

[4] King, M. N. (2004), (the Internet). Medical Biochemistry search, IU School of Medicine.

[5] Youngson, R. (2001). The ROYAL SOCIETY of MEDICINE, HEALTH Encyclopedia, Bloomsbury Publishing Plc. London.

[6] Morris, N. M. (1991). MASTERING ELECTRICAL ENGINEERING, THE MACMILLAN PRESS LTD.

[7] Medical Editor: Smith, T. (2000). Complete Family Health Guide, The British Medical Association, Dorling Kindersley limited, London.

[8] Green, N.P.O., Stout, G.W. and Taylor, D.J. (1990). Biological Science $1 \& 2$, Cambridge University Press.

[9] Fink, H.W. (2000). Electrical DNA, Press Conference for the American Physical Society. March 20-24.

[10] Fink, H.W. and Schonenberger, C. (1999). Electrical conduction through DNA molecules. Nature, 398, 407-410.

[11] Netherlands Organisation for Scientific Research. (Sep. 11, 2002). Report titled: DNA's oscillating double helix hinders electrical conduction.

[12] Porath, D., Bezryadin, A., de Vries, S. and Dekker, C. (2000). Direct measurement of electrical transport through DNA molecules. Nature, 403, 635.

[13] Bharadwaj, L.M., I. Kaur, I., Kumar, R. and Bajpai, R.P. 2000a. Design simulation of DNA based electronic components. Proc. SPIE, 4937, pp. 319-325.

[14] Asai, Yoshihiro, (2003), Small polaron model for electric current through single DNA molecule. J. Phys. Chem, B 107, 4647.

[15] Yi, J., and Orland, H. (Oct. 2004), Electric response of DNA hairpins to magnetic field. Cite Base (autonomous citation navigation and analysis.

[16] Cohn, H., Nogues, C., Naaman, R. and Porath, D. (2005), Direct measurement of electrical transport through single DNA molecules of complex sequence. Proceedings of the National Academy of Sciences of the United State of America (2005), Volume: 102, Issue: 33, Publisher: National Academy of Sciences, PP: 11589 - 11593.

[17] Jun Qian, Jun Qian, Sicheng, Liao Sicheng, Liao Stroscio, M.A. , Dutta, M. ,Song Xu, Song Xu , (2009), Electrical Transport through Single DNA Molecules by Distinct TipSurface Configurations, 2009 13th International Workshop on Computational Electronics, ISBN: 9781424439256, DOI 10.1109/IWCE.2009.5091111 
[18] Takagi, S., Takada, T. Matsuo, N. , Yokoyama, S., Nakamura, M. and Yamana, K. , (2012), Gating electrical transport through DNA molecules that bridge between silicon nanogaps. Nanoscale (2012) Volume: 4, pp: 1975-1977 ST - Gating electrical transport throug. ISSN: 20403364, DOI: $10.1039 / \mathrm{c} 2 \mathrm{nr} 12106 \mathrm{a}$

[19] Nunez, M.E., Hall, D.B. and Barton, J.K. (Feb. 1999). Longrange oxidative damage to DNA: effect of distance and sequence. Chemistry \& Biology 6 (2): pp. 85-97.

[20] Montagnier, L. , Aissa, J ,Ferris, S. , Montagnier, J. L. , Lavall'ee, C. , (2009), Electromagnetic Signals Are Produced by Aqueous Nanostructures Derived from Bacterial DNA
Sequences. Interdisciplinary Science: Computational Life science, DOI: $10.1007 / \mathrm{s} 12539$ - 009 - 0036 - 7

[21] Montagnier, L., Aissa, J., Del Giudice , E., Lavallee, C., Tedeschi, A and Vitiello, G., DNA waves and water, $2011 \mathrm{~J}$. Phys.: Conf. Ser. 306012007

[22] Williams, J. E., Metcalfe, H.C., Trinklein, F.E. and Lefler, R.W. 1968. Modern Physics, Holt, Rinehart and Winston, Inc.

[23] Editors: Bisacre, M., Carlisle, R., Robertson, D. and Ruck, J. 1979. The Marshall Cavendish Illustrated Encyclopedia of SCIENCE AND TECHNOLOGY, Marshall Cavendish Books Ltd. 